\title{
Assessing Catalytic Rates of Bimetallic Nanoparticles with Active Site Specificity - A Case Study using NO Decomposition
}

\author{
Joakim Halldin Stenlid ${ }^{[1],[2] \#}$, Verena Streibel ${ }^{[1][2] \#}$, Tej S. Choksi ${ }^{[1][2][3]}{ }^{*}$ and Frank Abild-Pedersen ${ }^{[1]^{*}}$
}

[1] SUNCAT Center for Interface Science and Catalysis, SLAC National Accelerator Laboratory, 2575 Sandhill Road, Menlo Park, California, 94025, United States.

[2] SUNCAT Center for Interface Science and Catalysis, Department of Engineering, Stanford University, 443 Via Ortega, Stanford, California, 94305, United States.

[3] School of Chemical and Biomedical Engineering, 62 Nanyang Drive, Nanyang Technological University, 637459, Singapore.

\# These authors contributed equally to this work.

*tej.choksi@ntu.edu.sg and abild@slac.stanford.edu

\begin{abstract}
Bimetallic alloys have emerged as an important class of catalytic materials, spanning a wide range of shapes, sizes, and compositions. The combinatorics across this wide materials space makes predicting catalytic turnovers of individual active sites challenging. Herein, we introduce the stability of active sites as a descriptor for site-resolved reaction rates. The site stability unifies structural and compositional variations in a single descriptor. We compute this descriptor using coordination-based models trained with DFT calculations. Our approach enables instantaneous predictions of catalytic turnovers for nanostructures up to $12 \mathrm{~nm}$ in size. Using NO dissociation as probe reaction, we identify that octahedral $\mathrm{Au}-\mathrm{Pt}$ core-shell nanoparticles and $3 \mathrm{~nm} \mathrm{Au}_{0.5} \mathrm{Pt}_{0.5}$ random alloys yield greater than 10 times higher compared to monometallic Pt nanoparticles. By prescribing specific sizes, morphologies, and compositions of optimal catalytic nanoparticles, our method provides tailored guidance to experiments for rationally designing bimetallic catalysts.
\end{abstract}

\section{INTRODUCTION}

Density functional theory (DFT)-derived Sabatier volcano plots have revolutionized the computationally guided discovery of transition metal catalysts. These plots correlate turnover frequencies (TOFs) to catalytic descriptors like the binding energies of reaction intermediates. ${ }^{[1-3]}$ The descriptors and reaction pathways are typically evaluated using ideal extended surfaces, which are simplified representations of nanoparticle facets. Real catalytic nanoparticles, especially compositionally flexible bimetallic alloys, however, are complex nanostructures spanning diverse structures and compositions, and exposing a distribution of active sites. This structural complexity results in a materials gap between nanostructures under reaction conditions and their idealized crystal plane models. This materials gap limits both experimental and computational evaluations of active site-resolved reaction rates. To bridge this gap, various structure-activity relationships have emerged for bimetallic surface facets, and more recently for bimetallic nanoparticles. ${ }^{[4-7]}$ Using features of active sites as inputs, both physics-based ${ }^{[4-13]}$ 
and data-driven ${ }^{[14-19]}$ relationships can evaluate catalytic descriptors with accuracy of 0.1 to $0.2 \mathrm{eV}$ compared to DFT. Applying these active site-resolved descriptors for nanoparticles holds promise for efficient predictions of nanoparticle size, shape, and composition yielding optimal reaction rates. Such specific knowledge provides tailored guidance for the synthesis of catalytic nanoparticles. It is still a challenge, however, to translate these descriptors into active site-resolved reaction kinetics across the distribution of active sites ubiquitous to bimetallic nanoparticles.

Herein, we introduce a generalizable framework for determining reaction rates and other kinetic metrics (e.g., rate orders) of bimetallic nanoparticles with active site resolution. Our approach leverages inherent correlations between the stability of active sites $\left(\overline{B E}_{M}\right)$ and their reactivity, as has been observed in experiments. ${ }^{[20,21]}$ We employ $\overline{B E}_{M}$ as a unified descriptor for both structural and compositional variations around active sites. This descriptor is predicted on-the-fly using coordinationbased models. ${ }^{[4-7]} \overline{B E}_{M}$ is then propagated through a family of local volcano plots to determine siteresolved reaction rates. Our general approach for evaluating site-resolved reaction rates is applicable in thermal and electrochemical catalysis. ${ }^{[22]}$ Previous efforts in describing catalytic activity of (bi)metallic nanoparticles include using semi-empirical methods, ${ }^{[23]}$ data-driven and machine-learning approaches, ${ }^{[17,19,24-30]}$ local $^{[31-33]}$ and global $^{[34,35]}$ physiochemical properties, and other modelling strategies..$^{[4,7,9,10,36-42]}$ Compared to existing methods, our approach is more readily generalizable across late transition metal alloys while maintaining an accuracy of $\pm 0.1-0.2 \mathrm{eV}$ in evaluating catalytic descriptors. ${ }^{[5,6]}$ Furthermore, our approach determines active site-resolved reaction rates for large systems (45 000 atoms) on the time scale of seconds to minutes while simultaneously accounting for catalytic activity and durability of an active site.

As benchmark system, we use the catalytic decomposition of $\mathrm{NO}(\mathrm{g})$ into $\mathrm{N}_{2}(\mathrm{~g})$ and $\mathrm{O}_{2}(\mathrm{~g})$ over Pt-based catalysts. This reaction is a technologically relevant probe reaction given its importance in purifying vehicle exhaust gases. ${ }^{[43-47]}$ Volcano plots on individual surface facets for NO dissociation have already been derived and used for catalyst screening. ${ }^{[48,49]}$ They show that Pt is located near the region showing maximum rate. Having these volcano plots as points of comparison makes NO dissociation over Pt-based catalyst an ideal platform to demonstrate how our scheme enables nanoparticle design through active site-resolved reaction rates. NO dissociation is shown as a four-step mechanism in equations (1) to (4), valid at the reaction conditions ( $\geq 700 \mathrm{~K})$ considered herein. ${ }^{[50]}$ Surface sites are indicated using *. $\mathrm{NO}(\mathrm{g})$ adsorption is followed by $\mathrm{NO}^{*}$ dissociation to $\mathrm{N}^{*}$ and $\mathrm{O}^{*}$, and finally the desorption of $\mathrm{N}_{2}(\mathrm{~g})$ and $\mathrm{O}_{2}(\mathrm{~g})$.

$$
\mathrm{NO}(\mathrm{g})+* \rightleftharpoons \mathrm{NO}^{*}
$$




$$
\begin{aligned}
& \mathrm{NO}^{*}+* \rightleftharpoons \mathrm{N}^{*}+\mathrm{O}^{*} \\
& 2 \mathrm{~N}^{*} \rightleftharpoons \mathrm{N}_{2}(\mathrm{~g})+2^{*} \\
& 2 \mathrm{O}^{*} \rightleftharpoons \mathrm{O}_{2}(\mathrm{~g})+2^{*}
\end{aligned}
$$

To demonstrate our approach, we evaluate active site-resolved reaction rates of NO decomposition on bimetallic Pt nanoparticles ranging from $147(1.6 \mathrm{~nm})$ to $43719(12 \mathrm{~nm})$ atoms. We find that nanoparticles of specific shapes (octahedral), sizes (2-3 nm), and compositions ( $\left.\mathrm{Au}_{0.5} \mathrm{Pt}_{0.5}\right)$ can increase rates by up to two orders of magnitude compared to monometallic Pt nanoparticles. By considering atomic-level and macroscopic kinetics on an equal footing, our method bridges the size gap in materials modeling, thus permitting direct computational studies of nanostructured catalysts.

\section{RESULTS AND DISCUSSION}

In brief, our method, outlined in Figure 1, is a stepwise link between the local structure of the active site and its catalytic activity. We evaluate the stability of the active site (henceforth referred to as the average binding energy of the site, $\overline{B E}_{M}$ ) using a coordination-based model. ${ }^{[4,7]}$ This site stability serves as input into a set of linear relations that yield binding energies of reaction intermediates. These binding energies are in turn used to estimate reaction barriers and activation energies of elementary steps at that active site. The active site-specific reaction energetics enter a microkinetic model that computes site-resolved reaction rates on the nanoparticle surface.

In our workflow, we first efficiently predict $\overline{B E}_{M}$ using a coordination-based model, which we term as the alloy stability model (ASM) (see Figure 1.A). This model is described in previous publications ${ }^{[4-6]}$ and summarized in supporting note $\mathbf{S 1}$ of the supplemental information (SI). In brief, for a given site ensemble composed of atoms $i$ of element $Z_{i}$ (e.g., $Z_{i}=\mathrm{Pt}$ ), of size $n$ (e.g., $n=2$ for bridge sites), and with nearest neighbors $j$ (e.g., $\left.Z_{j}=\{\mathrm{Pt}, \mathrm{Au}\}\right)$ the binding energy $\overline{B E}_{M}(n, i, j)$ per atom is:

$$
\overline{B E}_{M}(n, i, j)=\frac{1}{n} f\left(\mathbf{C N}_{i}, \mathbf{C N}_{j}, \mathbf{Z}_{i}, \mathbf{Z}_{j}\right)=\frac{1}{n} \mathbf{A} \cdot \boldsymbol{\alpha}
$$

The only inputs to this surrogate model in equation (5) are the coordination numbers $\left(\mathbf{C N}_{\mathbf{i}}, \mathbf{C N}_{\mathbf{j}}\right)$ and composition $\left(\mathbf{Z}_{\mathbf{i}}, \mathbf{Z}_{\mathbf{j}}\right)$ of the site ensemble as well as its nearest neighbors. The parameters of the alpha vector, $\boldsymbol{\alpha}$, denoted $\alpha_{\mathrm{N}, \mathrm{Z}}$ with $\mathrm{N}=\{1-12\}\left(\alpha_{1, \mathrm{Z}}\right.$ to $\alpha_{3, \mathrm{Z}}$ are grouped as $\left.\alpha_{1-3, \mathrm{Z}}\right)$ for fcc metals, reflect the average differential energy gained by an atom as it forms the $\mathrm{N}^{\text {th }}$ bond. The matrix $\mathbf{A}$ represents the changes in coordination number of atoms during the adsorption of the active site into the nanoparticle. 
By training the $\alpha_{\mathrm{N}, \mathrm{Z}}$ parameters against representative DFT calculations on simple surfaces, MAEs of $\pm 0.1 \mathrm{eV}$ versus DFT are obtained for Pt-based alloys. ${ }^{[6,7]}$ Evaluating active site stabilities using this scheme is central to our approach, enabling the examination of reactivity trends on nanostructures up to $12 \mathrm{~nm}$ in size ( 45 000 atoms). While we only consider Pt sites in this work, the methods are generalizable to any kind of metal alloy site. ${ }^{[6]}$

Next, we leverage linear site-specific scaling relations between $\overline{B E}_{M}$ and the adsorption energy $\left(\Delta E_{\text {ads }}\right)$ of reaction intermediates onto that site (e.g., $\left.\mathrm{N}^{*}, \mathrm{O}^{*}, \mathrm{NO}^{*}\right){ }^{[6]}$ see Figure 1.B and supporting note $\mathbf{S 2}$. Such relations between stability and reactivity for transition metal nanoparticles are inferred through experiments. ${ }^{[20,21]}$ The $\Delta E_{\text {ads }}$ of key reaction intermediates are connected to the activation energy $\left(E_{\mathrm{a}}\right)$ of elementary steps (herein, eq. 1-4) through Brønsted-Evans-Polanyi (BEP) relations (Figure 1.B and supporting note S3). ${ }^{[48,51,52]}$ We add entropic changes to the estimated barriers and reaction energies to compute free energy changes and site-specific rate constants (supporting note S4). These rate constants are inputted into a mean-field microkinetic model (supporting note S6).

Classical volcano plots based on mean-field microkinetic models represent reaction rates for a given surface facet in terms of binding energies of reaction intermediates. ${ }^{[2,53]}$ To provide a more realistic model for nanoparticles, exposing multiple surface facets, instead of using a single volcano plot, our approach introduces a family of local volcano plots. These plots efficiently sample site-specific reaction rates across diverse active sites in bimetallic nanoparticles. We create local volcano plots for sites having a specific composition (e.g., Pt) and a specific structure (e.g., 7-7 coordinated bridge sites) using $\overline{B E}_{M}$ as the sole descriptor (Figure 1.C). The $\overline{B E}_{M}$ directly maps variations in chemical composition around the active site to the local TOF. Finally, under the assumption that each site operates autonomously without being influenced by neighboring sites, the total rate for a given nanoparticle size and shape is computed as the sum of contributions from individual sites (Figure 1.D). We discuss the validity of the autonomous site assumption after presenting our results. With our framework, we can zoom into promising regions of existing volcano plots and identify precise shapes, sizes, and compositions of bimetallic nanoparticles yielding optimal rates. We now leverage our methods illustrated in Figure 1 to design nanocatalysts for NO decomposition. The approach delineated in Figure 1 is generalizable to other thermal and electrocatalytic reactions as long as parameters like $\boldsymbol{\alpha}_{\mathrm{N}, \mathrm{Z}}$ as well as slopes and intercepts of linear fits are available, in addition to a general understanding of the reaction mechanism. 


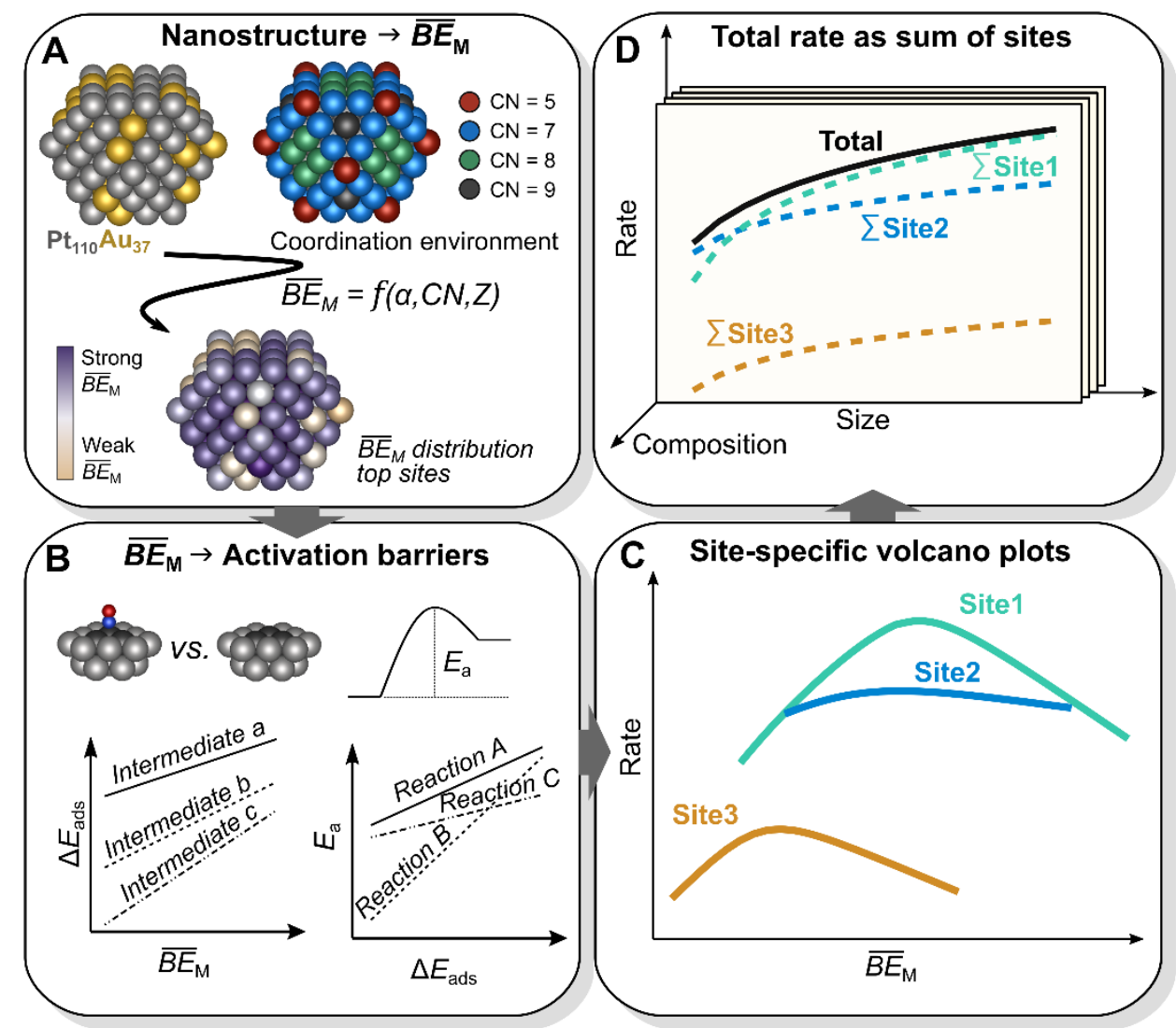

Figure 1. Stepwise approach for predicting active site-specific reaction rates on nanoparticles. In $\mathbf{A})$, we use the alloy stability model $^{[4-6]}$ to compute the binding energies of active sites $\left(\overline{B E}_{M}\right)$. B) The $\overline{B E}_{M}$ in turn yields adsorption energies $\left(\Delta E_{\text {ads }}\right)$ of reaction intermediates through site-specific scaling relations. These site-specific $\Delta E_{\text {ads }}$ are inserted into Brønsted-EvansPolanyi relations to estimate activation energies $\left(E_{\mathrm{a}}\right)$ at every site. C) $\Delta E_{\text {ads }}$ and $E_{\mathrm{a}}$ are inputted into microkinetic models that yield local volcano plots expressing rates using $\overline{B E}_{M}$ as the only descriptor. D) The total reaction rate for the nanostructure is computed as the sum of contributions from each type of site.

We assume that the nanostructured surface contains active sites resembling structural motifs on extended surfaces. Falsig et al. ${ }^{[48]}$ studied NO decomposition on multiple surface facets of different transition metals, including Pt. Favored adsorption sites were evaluated on several facets, which included terraces, like (111) and (100) surfaces, and edges, like on (211) or (110) surfaces. Sites of special importance for NO decomposition were identified as the 3-fold fcc hollow sites on (111)-like surface motifs, and 2-fold bridge/step sites on (100)-like motifs or $(211) /(110)$ edges. $^{[48]}$

These facets and sites are found on common nanoparticles (NPs), e.g., NPs with octahedral morphologies. This family of shapes includes the perfect octahedral (OCTA), cuboctahedral (CUBO), and truncated octahedral particles. We find that truncated octahedral shapes are frequently formed in Wulff constructions (WULFF) in vacuum (supporting note 5). The OCTA, CUBO, and WULFF morphologies form the basis of our analysis.

Figure 2 shows the variation in $\overline{B E}_{M}$ with shape, size, and alloying pattern for different types of Pt sites on pure and alloyed Pt NPs. The $\overline{B E}_{M}$ are calculated using equation 5 . The sites are 
denoted by the coordination number $(\mathrm{CN})$ of the atoms constituting the site ensembles: e.g., the hollow fcc site on the (111) facets is denoted 9-9-9, whereas the bridge sites on (100) and the (211)/(110) steps are referred to as 8-8 and 7-7, respectively. Prior studies on NO dissociation indicate that reaction rates on Pt are higher than on other monometallic systems or their alloys. Based on these insights, we assume that NO dissociation occurs only on pure Pt dimer and trimer ensembles in our binary alloys. Effectively, we thus assume that any site ensembles containing a non-Pt atom have negligible TOFs compared to pure Pt ensembles.

For pure Pt particles, the $\overline{B E}_{M}$ of a given type of site with a specific $\mathrm{CN}$ varies based on the number of next-nearest neighbors. This variation of the site stability is generally small and falls within an energy window of $\pm 0.3 \mathrm{eV}$, regardless of the particle shape and size (Figure 2.A-C). Given the small variations in $\overline{B E}_{M}$ for monometallic Pt particles, the reaction rates per particle are mainly influenced by the number of active sites in different morphologies. In Figure 2.G; if the 9-9-9 site dominates the total catalytic activity, OCTA is the preferred catalyst shape, whereas if the 8-8 sites are more active, CUBO is a better choice. Further details on size, shape, and structure effects are provided in the supporting note S7.

Alloying has a larger effect on the $\overline{B E}_{M}$ than changing the number of next-nearest neighbors of a monometallic system. With appropriate binary combinations, the $\overline{B E}_{M}$ of a $\mathrm{Pt}_{3}$ 9-9-9 site is destabilized by $0.83 \mathrm{eV}$ in an $\mathrm{Au}$ environment and stabilized by $0.86 \mathrm{eV}$ when surrounded by $\mathrm{Ir}$ (supporting note S8). Figure 2.D-F demonstrate the effects of systematically and randomly alloying Pt particles with Au. We will show in Figure 3 and in supporting note S9 that these alloys enhance the rates of NO dissociation. The higher intrinsic rates per site, however, come at the cost of deactivating other sites that directly incorporate inert $\mathrm{Au}$ atom(s). These tradeoffs are discussed below in connection with Figure 5. 

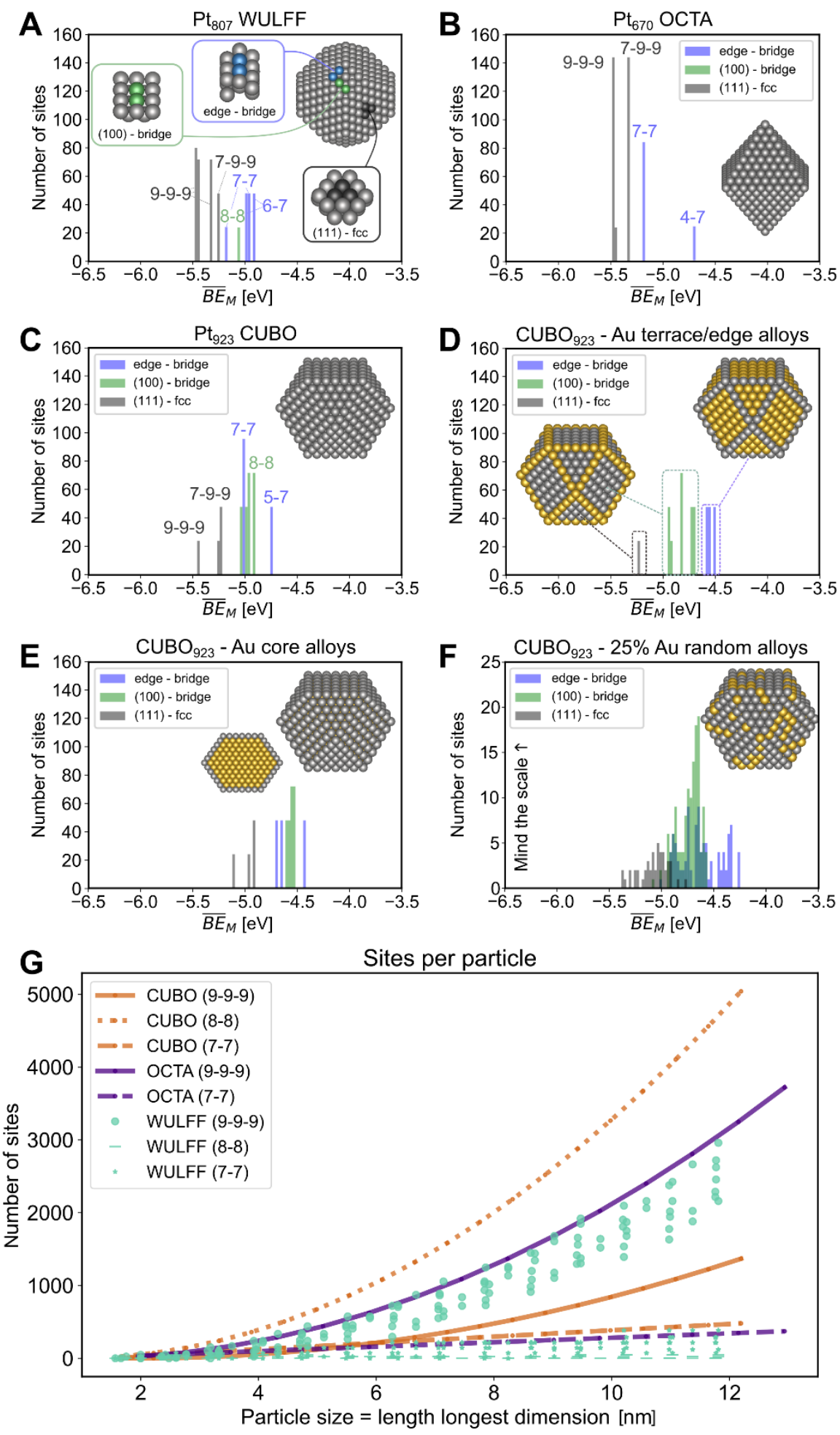

Figure 2. A)-C), distribution of sites and associated $\overline{B E}_{M}$ over nanoparticles of different shapes. $\overline{B E}_{M}$ is normalized by the number of atoms in the site, e.g., 2 for bridge sites. D)-F), distributions of sites for different Au alloying patterns. Distributions for sites relevant to NO decomposition are shown. In A)-C), the coordination number (CN) of the atoms of the sites are marked. G) reports the size-evolution of the number of sites for different nanoparticle shapes. The longest dimension refers to the largest atomic distance within the particle. Considered shapes include octahedral (OCTA), cuboctahedral (CUBO) and Wulff constructions (WULFF). For WULFF, we generate particles based on a finely discretized size range as described in Supporting note 5. Note that the 3-fold hollow 9-9-9 and 7-9-9 sites refer to fcc sites. 
We now link the site stability $\overline{B E}_{M}$ to its catalytic activity via the procedure outlined in Figure 1. This link allows us to create volcano plots like the one shown in Figure 3 for NO decomposition on 9-9-9 Pt sites at $700 \mathrm{~K}, 10 \mathrm{mbar} \mathrm{NO}(\mathrm{g})$, and 5\% conversion. These site-specific volcano plots correlate reaction rates at a given metal site (e.g., Pt) having a specific structure (e.g., 9-99 fcc sites) with variations in the chemical composition around the site (supporting note S6). To illustrate the widest range of composition effects on $\mathrm{NO}$ decomposition rates, we label Pt ensembles surrounded by different metals (e.g, Au, Ir, etc.) in Figure 3. These variations can to the first order be described by a shift of the upper band edge of the d-states, as discussed elsewhere. ${ }^{[54]}$ In Figure 3, the $\overline{B E}_{M}$ of pure $\mathrm{Pt}$ is $\sim 1.2 \mathrm{eV}$ too negative compared to the volcano peak. As we observe, however, alloying Pt with, e.g., Au can be used to render $\overline{B E}_{M}$ more positive and shifting the ensemble closer to the volcano peak. In fact, surrounding Pt ensembles with Au increases the intrinsic rate by two orders of magnitude. Similar volcano plots for 7-7 bridge sites located at nanoparticle edges and 8-8 bridge sites on (100) facets are shown in supporting note 6. Since $\overline{B E}_{M}$ can be predicted using the computationally efficient alloy stability model (ASM), we can leverage such site-specific volcano plots to instantaneously evaluate rates at $\mathrm{Pt}$ sites surrounded by late transition metals on nanoparticles of $>10 \mathrm{~nm}$ size.

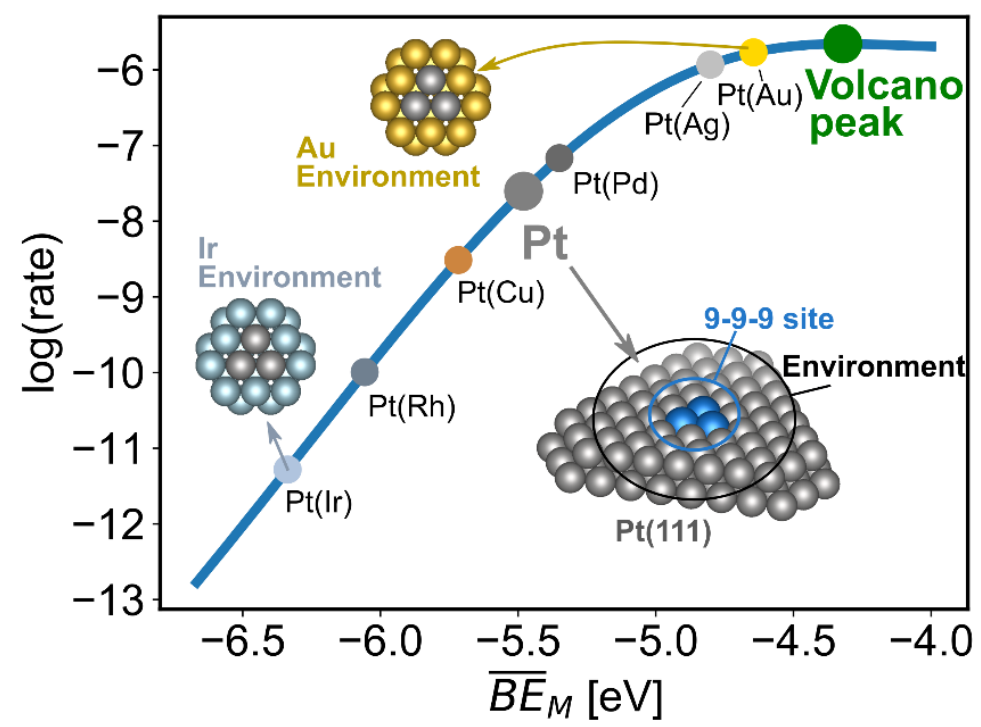

Figure 3. An example of a local volcano plot for NO dissociation at 9-9-9 coordinated fcc sites. Rates are expressed as a function of the $\overline{B E}_{M}$ of the site. The $\overline{B E}_{M}$ can be modified by varying the chemical environment around the site, for instance surrounding the $\mathrm{Pt}_{3} \mathrm{fcc}$ site with $\mathrm{Ir}, \mathrm{Rh}, \mathrm{Cu}, \mathrm{Pd}, \mathrm{Ag}$, and $\mathrm{Au}$. Reaction rates are computed at $700 \mathrm{~K}, p_{\text {tot }}=1 \mathrm{bar}, p_{\mathrm{NO}}=10 \mathrm{mbar}$, $5 \%$ conversion (supporting note 6). Similar local volcanoes for 7-7 sites at step edges and 8-8 sites on (100) terraces are shown in supporting note 6. Note that opposite to traditional volcano plots, we switch from low to high coverage as we move from the left (more negative $\overline{B E}_{M}$ ) to right (less negative $\overline{B E}_{M}$ ). This reversed trend is because $\overline{B E}_{M}$ is inversely correlated with the adsorption energies of reaction intermediates (supporting note 2 ).

In addition to accelerating the evaluation of site-resolved rates, $\overline{B E}_{M}$ also explains trends in kinetic metrics ${ }^{[55-58]}$ of NO decomposition like degrees of kinetic/thermodynamic control, ${ }^{[56-58]}$ rate 
orders, coverages, and apparent activation energies. These trends on 9-9-9 Pt sites as a function of their chemical composition are shown in Figure 4.A-F. Thus, changes in mechanistic aspects of chemical reactions can be expressed using $\overline{B E}_{M}$ as the only variable. The degree of kinetic control in Figure 4.A indicates that based on $\overline{B E}_{M}$, two mechanisms are followed. At more negative $\overline{B E}_{M}$, the N-N bond formation has a degree of rate control of 1, thus being the sole rate-determining step. At less negative $\overline{B E}_{M}$, the degree of kinetic control for both O-O formation and $\mathrm{N}-\mathrm{O}$ dissociation increases at the expense of N-N dissociation. The volcano plot in Figure 3 has a relatively flat maximum because of the competing influences of three elementary steps in the pathway. At more negative $\overline{B E}_{M}$, the surface has a high coverage of empty sites (Figure 4.B), which is further elucidated by the negative degree of thermodynamic control for empty sites (Figure 4.C). Upon shifting to less negative $\overline{B E}_{M}$, Pt ensembles exhibit higher coverage of $\mathrm{NO}^{*}$ and $\mathrm{O}^{*}$ together with lower rate orders for $\mathrm{NO}(\mathrm{g})$ and $\mathrm{O}_{2}(\mathrm{~g})$ (Figure 4.D). As $\overline{B E}_{M}$ become less negative, both the reaction rate and the apparent activation energy (Figure 4.E) increase. Seemingly counter-intuitive, the reaction rate increases because any increases in the apparent activation energy are more than compensated for by an increase in the apparent pre-factor (Figure 4.F). This phenomenon, known as the chemical compensation effect, ${ }^{[55]}$ arises due to changes in the reaction mechanism on either side of the volcano plot. A longer discussion about mechanistic details is included in the Supporting note S6. Figure 4.A-F quantitatively shows how the fundamental kinetic properties of a reaction are inherently governed by the stability of catalysts, as has been inferred in experiments. ${ }^{[20,21,59]}$

We now discuss the assumptions used for further analysis on nanoparticles. First, only pure Ptensembles in Pt-bimetallic particles contribute to the reaction rate. Second, the reaction rate of 9-9-9 Pt sites dominates over other coordinated sites (e.g., 7-7 or 8-8). Third, these 9-9-9 sites operate autonomously. The first assumption is appropriate since previous studies have shown that pure $\mathrm{Pt}$ has the highest rates for NO dissociation with the rates on other metals being orders of magnitude lower. ${ }^{[48,49]}$ The second assumption is valid since the site-specific volcano plots in our work indicate that 9-9-9 terrace sites have higher rates $\left(10^{-7}\right.$ to $\left.10^{-6} \mathrm{~s}^{-1}\right)$ than both 7-7 bridge sites $\left(10^{-12}\right.$ to $\left.10^{-11} \mathrm{~s}^{-1}\right)$ and $8-8$ terrace sites $\left(10^{-18}\right.$ to $\left.10^{-15} \mathrm{~s}^{-1}\right)$ at $700 \mathrm{~K}$. Moreover, some experimental reports hint that 9-9-9 rich $\mathrm{Pt}(111)$ facets are more active than other facets in NO decomposition (see supporting note 10). ${ }^{[60,61]}$ These 9-9-9 sites balance between $\mathrm{N}-\mathrm{O}$ bond activation and the desorption of $\mathrm{N}^{*} / \mathrm{O}^{*}$ as $\mathrm{N}_{2}(\mathrm{~g})$ and $\mathrm{O}_{2}(\mathrm{~g})$. Our third assumption about autonomous sites is reasonable because both our microkinetic model on 9-9-9 Pt ensembles (coverage of free sites, $\Theta^{*},>0.25$ except near the volcano maximum) and previous studies ${ }^{[48]}$ on $\mathrm{Pt}(111)$ surfaces $\left(\Theta^{*} \approx 0.5\right.$ in the region around $\left.\mathrm{Pt}\right)$ indicate that the coverage of free sites is 
comparatively high. Thus, for a large range of $\overline{B E}_{M}$, the higher coverage of free sites on 9-9-9 leads to negligible adsorbate-co-adsorbate effects and vanishing cooperative/inhibiting effects of neighboring sites. These aspects lend credibility to the autonomous site assumption for the 9-9-9 sites. This approximation is, however, less valid near the volcano peak and future studies should address coadsorbate effects for increased accuracy.
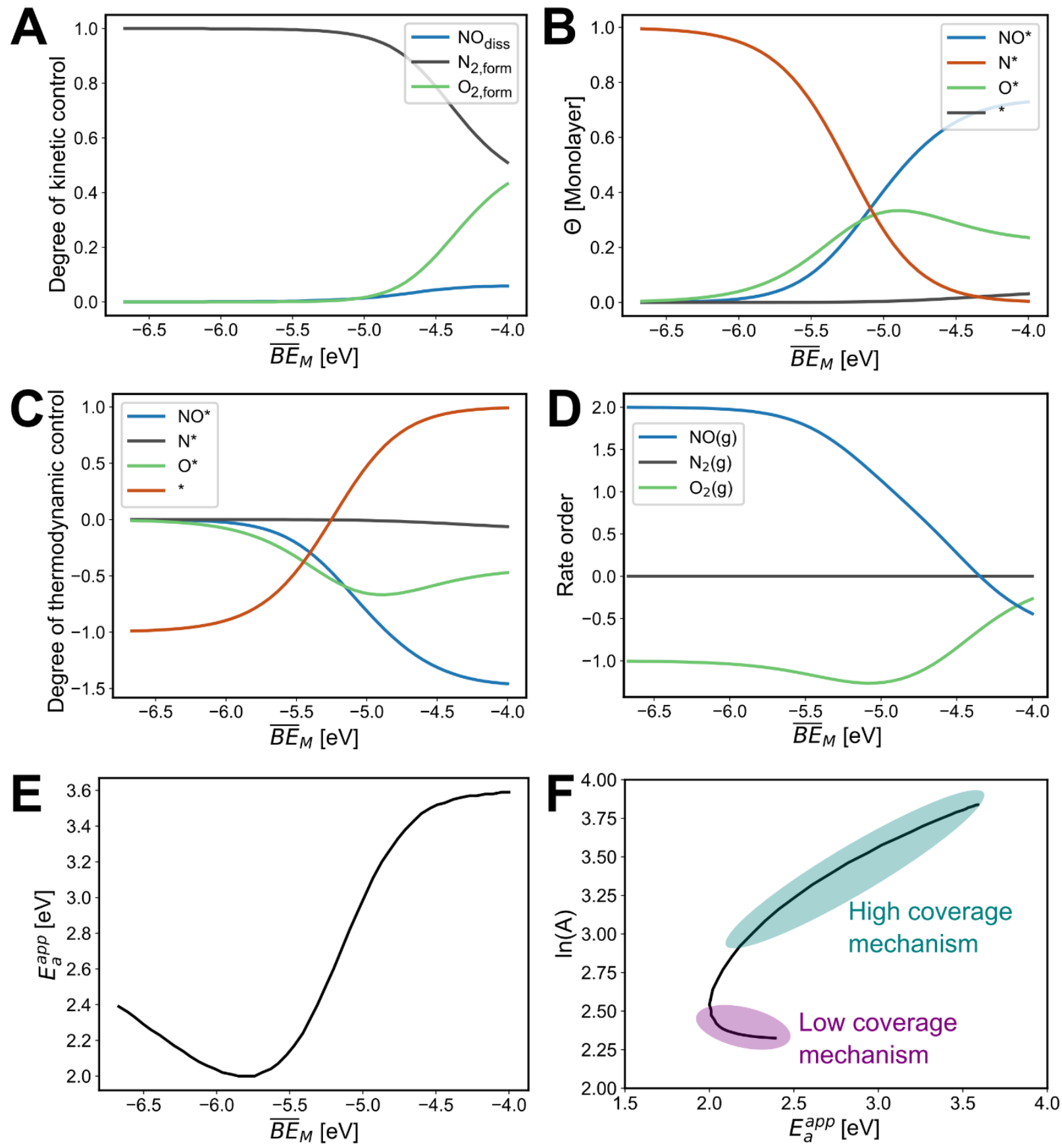

Figure 4. The site stability, $\overline{B E}_{M}$, systematizes trends in kinetic metrics like the degree of kinetic control (A), coverage of surface species $(\mathbf{B})$, degree of thermodynamic control $(\mathbf{C})$, rate orders $(\mathbf{D})$, and the apparent activation energy $\left(E_{a}^{a p p}\right)(\mathbf{E})$. We evaluate these kinetic metrics for 9-9-9 Pt sites as chemical environment around the site is varied. $E_{a}^{a p p}$ compared to $\ln (\mathrm{A})$, with A being the apparent kinetic prefactor, in $\mathbf{F}$ highlighting two distinct mechanistic regions corresponding to high and low coverage. A full monolayer in (B) corresponds to every site being occupied. 
Based on the assumptions, we highlight the versatility of this framework in determining site-resolved rates across both pure Pt and Pt-Au bimetallic nanoparticles in Figure 5. Pt is alloyed with $\mathrm{Au}$ as this combination yields the largest shift towards the $\overline{B E}_{M}$ of the volcano peak (Figure 3). We note, however, that Ag has a similar effect. Depending on, e.g., economical, synthesis, or durability arguments, the ideal catalysts might thus be generated using other alloying metals than Au. Discussion and results on other alloys are included in supporting note $\mathbf{S 9}$.

We first assess monometallic Pt particles. Figure 5.A-D shows the rates for WULFF, OCTA, and CUBO nanoparticles presented per surface area, per particle, per Pt atom, and per 9-9-9 site (metrics explained further in supporting note 11). In general, OCTA is the most active nanoparticle shape regardless of the metric. Specific WULFF shapes having higher densities of (111) facets can yield metrics comparable to OCTA. In general, however, WULFF particles have lower reactivity metrics because unlike OCTA, these particles contain less reactive sites such as the 8-8 sites on (100) facets. For all shapes, the rate per surface area is comparably low for small particles and increase asymptotically with size (Figure 5.A). The rate per particle also increases with size (Figure 5.B) as the larger particles possess higher densities of 9-9-9 sites. However, to optimize the used amount of the precious Pt metal, the most relevant metric is the rate normalized by the number of Pt atoms in the catalyst. In case of all particle shapes, this metric yields a clear peak in performance as shown in Figure 5.C; OCTA peaks at diameters of $\sim 3 \mathrm{~nm}$ whereas CUBO peak at diameters $\sim 5 \mathrm{~nm}$. Comparing the rates at the performance peaks, OCTA is between 2.5 and 5 times more active per Pt atom than the other shapes (using the average value of WULFF), due to its higher density of 9-9-9 sites. Since the rate per site (Figure 5.D) is approximately constant for the pure Pt particles, the changes in the rate per area (or per particle) follow the increase in coverage (number) of 9-9-9 sites with size. Eventually the per area rates converge to the value of the extended (111) facet weighted by the prevalence of the facet for the given nanoparticle shape.

Alloying with Au can further increase the activity of the particles by more than an order of magnitude, as shown in Figure 5.F-H exemplified by the OCTA particles. As for the pure particles, the rates per particle of the PtAu alloys increase continuously with size as the number of exposed 9-9-9 sites increases (Figure 5.F). Systematically alloying the edges or core with $\mathrm{Au}$ improves the activity significantly, with AuPt core-shell particles displaying the largest activity of all considered cases - except for the smallest particles of $\sim 2 \mathrm{~nm}$ size where Au edge alloys are more active (see discussion in supporting note 9). For randomly alloyed NPs, the largest improvement in activity is seen when alloying with $50 \% \mathrm{Au}$ demonstrating the trade-off between activating some Pt sites through alloying in the first coordination sphere, while inhibiting other sites through incorporation of the inert Au atoms. Using the 
rate per Pt atom metric (Figure 5.G), we find the same ordering as above for the activity of particle types; AuPt core-shell particles are displaying the largest rate for most sizes and the $\mathrm{Pt}_{0.5} \mathrm{Au}_{0.5}$ alloys have the best performance of the random alloys. For the random alloys, a performance peak at around $\sim 3 \mathrm{~nm}$ is seen, i.e., the same position as for the pure Pt OCTA particles. For AuPt core shell particles the rate increases monotonically with particle size. This monotonic increase occurs because all Pt atoms, per definition, are at the surface for the core-shell alloys, thus the activity increases with size following the same trend as the rate per surface area for the pure particles in Figure 5.A. Hence, we conclude that, by using the rate per Pt atom metric alone, the optimal catalysts would be Au-Pt core-shell nanoparticles containing an Au core as large as practicable, while maintaining high surface area. Further insight of the increased activity of the PtAu alloys can be gained from Figure 5.H using the activity per site (i.e., the TOF). Here it is clear that the 9-9-9 sites of bimetallic Pt-Au are more active than the 9-9-9 sites of pure Pt NPs. The most active sites are found on the random particles with $75 \% \mathrm{Au}$ alloying, which lie close to the maximal activation of Pt 9-9-9 sites in a pure Au environment. These highly alloyed particles will, nonetheless, display a lower rate per particle and per Pt atom than the other alloys because of a lower number of active sites. These trends reiterate the conclusion that the most active catalyst particles will be the ones balancing the trade-off between having highly activated alloyed sites while minimizing inhibition of other sites by extensive Au surface alloying. Through our method, we can design nanoparticles by quantitatively evaluating these tradeoffs between intrinsic rates and densities of active sites. 
Pure Pt

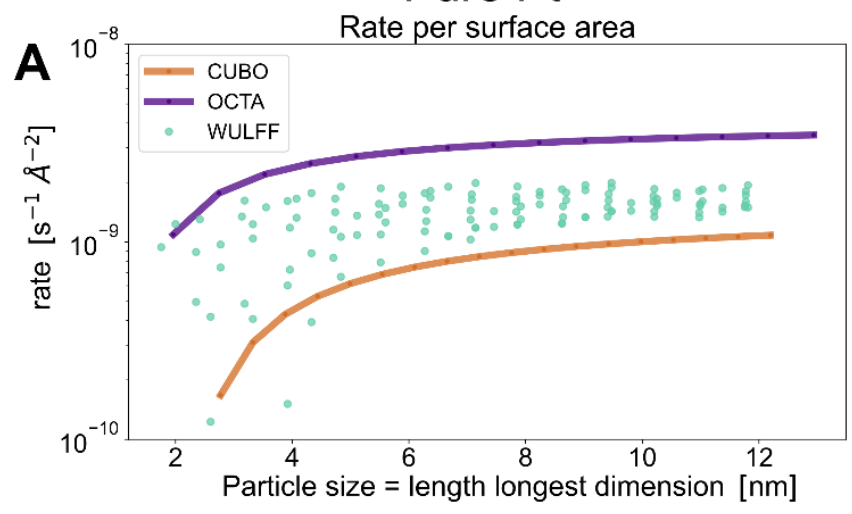

B

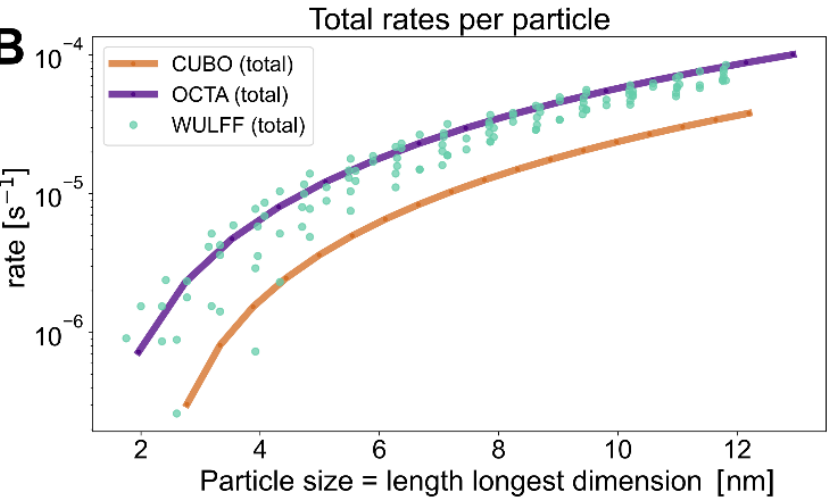

Rate per Pt atom

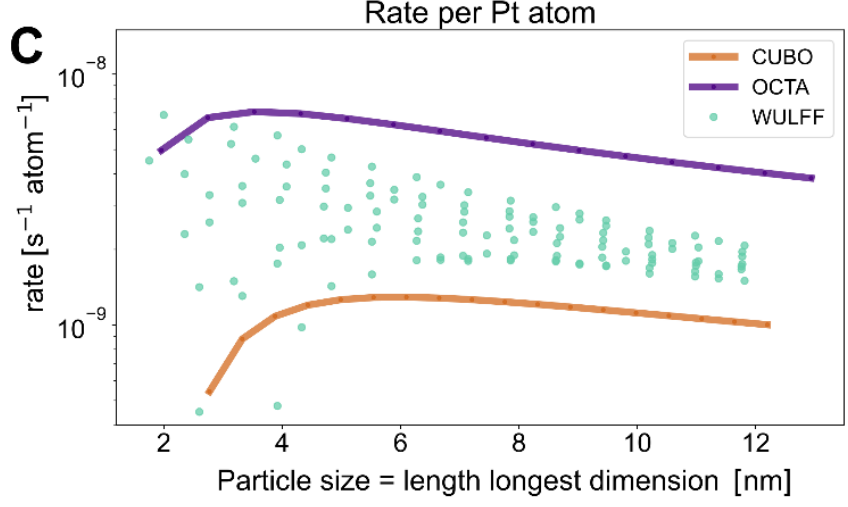

Rate per site

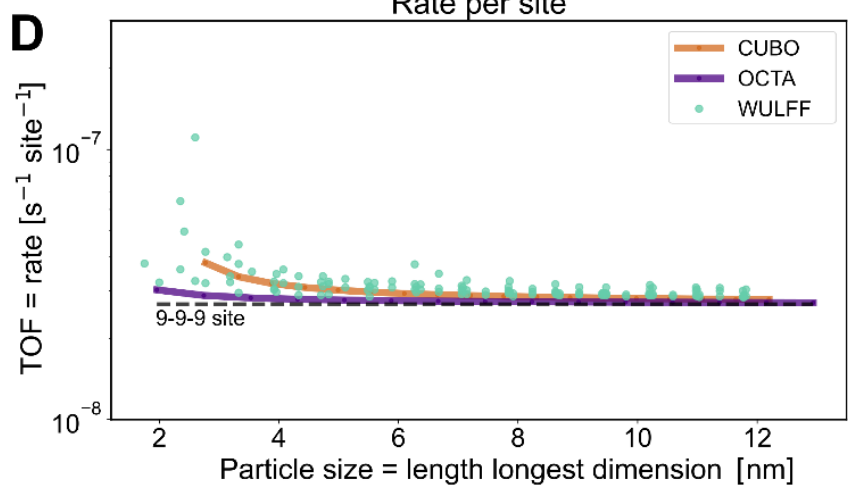

\section{PtAu alloys}
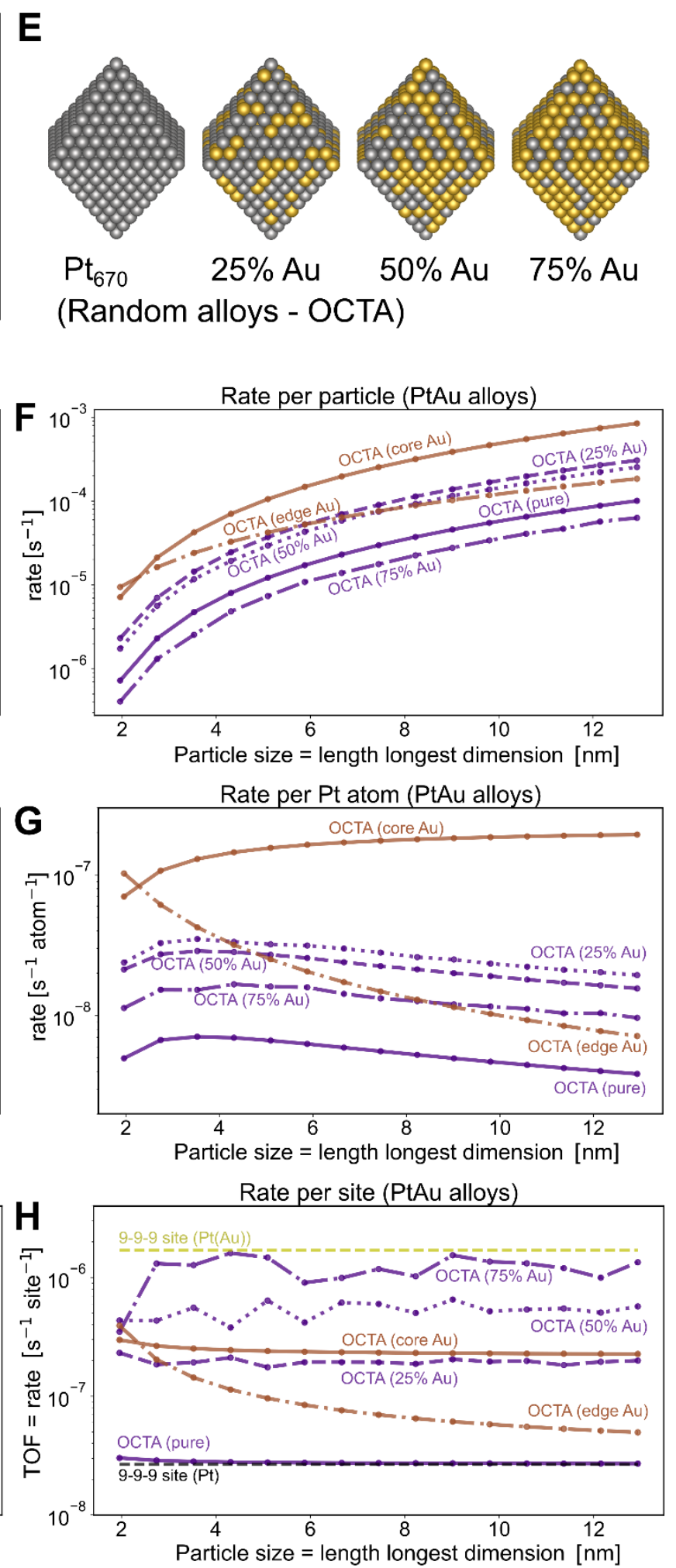

Figure 5. Predicted rates of NO decomposition for different nanoparticle shapes, sizes, and compositions assuming that the 9-9-9 site of $\mathrm{Pt}$ is the dominant active site. These rates can increase 100-fold compared to Pt by choosing appropriate alloying (AuPt core-shell), size (2-3 nm for non-core-shell particles), and shape (OCTA) for the catalysts nanoparticles. In A)-D), rates are computed per surface area (see supporting note 11), per particle, per Pt atom and per 9-9-9 site for pure Pt particles. E), examples of randomly alloyed PtAu OCTA particles. F)-G) rates per Pt atom, per particle, and per 9-9-9 site as a function of size and Au composition. To account for effects of segregation, rates are averaged over 100 randomly selected PtAu particles 
at a given size and composition. As an example, the rates for the random alloys in $\mathbf{F}$ have a standard deviation that increases from 1e-6 to $1 \mathrm{e}-5 \mathrm{~s}^{-1}$ site $^{-1}$ from the smallest to the largest particles. For WULFF, we generate a distribution of particles as described in supporting note 5.

Finally, we compare our rates per active site at different temperatures with those estimated in other theoretical studies ${ }^{[48]}$ and measured in experiments. ${ }^{[0,60-62]}$ At a lower temperature of $700 \mathrm{~K}$, relevant to application in exhaust gas converters for vehicles, our model-predicted rates on 9-9-9 Pt ensembles are 4-5 orders of magnitude lower than those measured in experiments. ${ }^{[50]}$ The rates computed in our work at $700 \mathrm{~K}$ are, however, within the same order of magnitude as previous mean-field microkinetic models for NO decomposition on Pt (111). ${ }^{[48]}$ At a higher temperature of $925 \mathrm{~K}$, our computed rates on 9-9-9 Pt ensembles are, instead, within 1-2 orders of magnitude of experiments (supporting note S10) ${ }^{[60-62]}$ Based on these two theory-experiment comparisons, we hypothesize that the larger difference between computation and experiments at $700 \mathrm{~K}$ is mainly due to the omission of adsorbate-co-adsorbate interactions in the models. At $925 \mathrm{~K}$, where we operate at low adsorbate coverages and hence negligible adsorbate-co-adsorbate interactions, experiment and theory are closely aligned.

A possibility to move the model closer to reality at lower temperatures, where adsorbateco-adsorbate interactions are important, ${ }^{[63-65]}$ is to account for spill-over effects from one site (e.g., N-O scission on step edges) to another (e.g., N-N recombination on terraces) by enabling site communication. Such communication requires a multiple-site model moving beyond the single-site mean-field approach used herein. ${ }^{[66,67]}$ For example, such a communicating multiple-site model could directly evaluate whether or not edge sites are poisoned by a high surface adsorbate coverage and are no longer available as adsorption sites. If such a coverage-dependence and inter-site communication are incorporated within the microkinetic model, our described methods remain valid with $\overline{B E}_{M}$ as the sole descriptor.

Taken together, we have demonstrated that our method efficiently provides active-siteresolved energetics and kinetic constants at low coverages for nanoparticles having arbitrary shapes, sizes, and compositions. If co-adsorbate effects and communications between sites is strong, these interactions can be added on to appropriately correct rate constants. Leveraging the inherent link between active site stability and its reactivity, our work serves as a generalized approach for the in-silico design of nanoparticles.

\section{CONCLUSION}

We present a method for evaluating active site-resolved reaction rates of nanostructures using the stability of active sites, $\overline{B E}_{M}$, as the sole descriptor. $\overline{B E}_{M}$ is, in turn, efficiently predicted using a 
coordination-based scheme. By propagating $\overline{B E}_{M}$ through a microkinetic model, our approach instantaneously probes site-specific catalytic properties with good accuracy compared to DFT for nanoparticles in the 2-12 $\mathrm{nm}$ size range. We have illustrated this strategy by designing bimetallic nanoparticles for a model reaction, NO decomposition. Our findings indicate that catalytic turnovers can be enhanced up to 100-fold by alloying Pt with Au (e.g., AuPt core-shell) and by choosing the optimal particle size (2-3 nm, for non-core-shell particles) and shape (octahedral particles). While our contribution focuses on the activity of monometallic sites in bimetallic environments for NO decomposition, we stress that our approach is generally applicable to evaluate the catalytic activity of mixed metal sites in various coordination and chemical environments. Hence, the methods introduced herein pave the way towards precisely tailoring the nanostructure of materials to design next-generation catalysts for metal-catalyzed processes.

\section{COMPUTATIONAL DETAILS}

All DFT calculations within this work were performed in Quantum ESPRESSO ${ }^{[68]}$ within the Atomic Simulation Environment. ${ }^{[69]}$ Total energies were determined using the RPBE functional, ${ }^{[70]}$ on k-point grids (Table S1) generated with the Monkhorst-Pack method. ${ }^{[71]}$ Atomic cores were represented by ultrasoft Vanderbilt pseudopotentials. ${ }^{[72]}$ A dipole correction was included for surface calculations. ${ }^{[73]}$ Further computational details are given in the SI. Atomic structures, energies, and scripts employed for analysis are uploaded to Catalysis-Hub ${ }^{[74]}$ (https://www.catalysishub.org/publications/HalldinAssessing2021) and Git-Hub (https://github.com/kjhstenlid/ASM_alphascheme) repositories.

\section{ACKNOWLEDGMENT}

This research was supported by the U.S. Department of Energy, Office of Science, Office of Basic Energy Sciences, Chemical Sciences, Geosciences, and Biosciences Division, Catalysis Science Program to the SUNCAT Center for Interface Science and Catalysis. J.H.S. and V.S. gratefully acknowledge financial support from the Knut and Alice Wallenberg Foundation (Grant No. 2019.0586) and the Alexander von Humboldt Foundation, respectively. T.S.C. was sponsored in part by the Ministry of Education Academic Research Fund Tier 1 seed grant (RS04/19). We acknowledge computational support from the National Energy Research Scientific Computing Center (Project Allocation No. m2997), a Department of Energy Office of Science User Facility supported by the Office of Science of the U.S. Department of Energy 
under Contract No. DE-AC02-05CH11231. The computational work for this article was partially performed on resources of the National Supercomputing Centre, Singapore (https://www.nscc.sg).

CONFLICT OF INTEREST

The authors declare no conflict of interest.

\section{KEYWORDS}

Catalytic descriptor; Heterogeneous catalysis; Nanostructures; NO decomposition; Platinum alloys

\section{REFERENCES}

[1] F. Abild-Pedersen, J. Greeley, F. Studt, J. Rossmeisl, T. R. Munter, P. G. Moses, E. Skúlason, T. Bligaard, J. K. Nørskov, Phys. Rev. Lett. 2007, 99, 016105.

[2] T. Bligaard, J. K. Nørskov, in Chemical Bonding at Surfaces and Interfaces (Eds.: A. Nilsson, L.G.M. Pettersson, J.K. Nørskov), Elsevier, Amsterdam, 2008, pp. 255-321.

[3] J. K. Nørskov, F. Studt, F. Abild-Pedersen, T. Bligaard, Fundamental Concepts in Heterogeneous Catalysis, John Wiley \& Sons, 2014.

[4] L. T. Roling, L. Li, F. Abild-Pedersen, J. Phys. Chem. C 2017, 121, 23002-23010.

[5] L. T. Roling, F. Abild-Pedersen, Chem CatChem 2018, 10, 1643-1650.

[6] T. S. Choksi, L. T. Roling, V. Streibel, F. Abild-Pedersen, J. Phys. Chem. Lett. 2019, 10, 18521859.

[7] L. T. Roling, T. S. Choksi, F. Abild-Pedersen, Nanoscale 2019, 11, 4438-4452.

[8] X. Ma, H. Xin, Phys. Rev. Lett. 2017, 118, 036101.

[9] F. Calle-Vallejo, J. I. Martínez, J. M. García-Lastra, P. Sautet, D. Loffreda, Angew. Chem. Int. Ed. 2014, 53, 8316-8319.

[10] J. Dean, M. G. Taylor, G. Mpourmpakis, Sci. Adv. 2019, 5, eaax5101.

[11] V. Streibel, T. S. Choksi, F. Abild-Pedersen, J. Chem. Phys. 2020, 152, 094701.

[12] T. S. Choksi, V. Streibel, F. Abild-Pedersen, J. Chem. Phys. 2020, 152, 094702.

[13] Y. Ding, Y. Xu, Y. Mao, Z. Wang, P. Hu, Chem. Commun. 2020, 56, 3214-3217.

[14] J. A. Esterhuizen, B. R. Goldsmith, S. Linic, Chem Catalysis 2021, 1, 923-940.

[15] O. Mamun, K. T. Winther, J. R. Boes, T. Bligaard, npj Comput Mater 2020, 6, 1-11.

[16] X. Ma, Z. Li, L. E. K. Achenie, H. Xin, J. Phys. Chem. Lett. 2015, 6, 3528-3533.

[17] R. Jinnouchi, R. Asahi, J. Phys. Chem. Lett. 2017, 8, 4279-4283.

[18] Z. Li, S. Wang, H. Xin, Nat Catal 2018, 1, 641-642.

[19] A. Palizhati, W. Zhong, K. Tran, S. Back, Z. W. Ulissi, J. Chem. Inf. Model. 2019, 59, 47424749.

[20] C. T. Campbell, Acc. Chem. Res. 2013, 46, 1712-1719.

[21] Z. Mao, C. T. Campbell, ACS Catal. 2021, 11, 8284-8291.

[22] J. A. Gauthier, J. H. Stenlid, F. Abild-Pedersen, M. Head-Gordon, A. T. Bell, ACS Energy Lett. 2021, 3252-3260.

[23] M. Núñez, J. L. Lansford, D. G. Vlachos, Nat. Chem. 2019, 11, 449-456.

[24] M. Andersen, K. Reuter, Acc. Chem. Res. 2021, 54, 2741-2749.

[25] R. Jinnouchi, H. Hirata, R. Asahi, J. Phys. Chem. C 2017, 121, 26397-26405.

[26] N. Artrith, A. M. Kolpak, Nano Lett. 2014, 14, 2670-2676.

[27] J. R. Boes, O. Mamun, K. Winther, T. Bligaard, J. Phys. Chem. A 2019, 123, 2281-2285.

[28] S. Hajinazar, J. Shao, A. N. Kolmogorov, Phys. Rev. B 2017, 95, 014114.

[29] S. Heiles, R. L. Johnston, Int. J. Quantum Chem. 2013, 113, 2091-2109. 
[30] A. Bruma, R. Ismail, L. O. Paz-Borbón, H. Arslan, G. Barcaro, A. Fortunelli, Z. Y. Li, R. L. Johnston, Nanoscale 2012, 5, 646-652.

[31] T. Brinck, J. H. Stenlid, Advanc. Theo. Catal. 2019, 2, 1800149.

[32] J. H. Stenlid, T. Brinck, J. Am. Chem. Soc. 2017, 139, 11012-11015.

[33] K. Saravanan, J. R. Kitchin, O. A. von Lilienfeld, J. A. Keith, J. Phys. Chem. Lett. 2017, 8, 50025007.

[34] L. Farsi, N. A. Deskins, Phys. Chem. Chem. Phys. 2019, 21, 23626-23637.

[35] E. M. Dietze, P. N. Plessow, F. Studt, J. Phys. Chem. C 2019, 123, 25464-25469.

[36] Z. Yan, M. G. Taylor, A. Mascareno, G. Mpourmpakis, Nano Lett. 2018, 18, 2696-2704.

[37] M. Jørgensen, H. Grönbeck, J. Chem. Phys. 2018, 149, 114101.

[38] E. M. Dietze, P. N. Plessow, J. Phys. Chem. C 2019, 123, 20443-20450.

[39] W. Chen, D. Schmidt, W. F. Schneider, C. Wolverton, J. Phys. Chem. C 2011, 115, 17915-17924.

[40] J. Cheng, P. Hu, Angew. Chem. Int. Ed. 2011, 50, 7650-7654.

[41] M. G. Taylor, N. Austin, C. E. Gounaris, G. Mpourmpakis, ACS Catal. 2015, 5, 6296-6301.

[42] S. Wang, N. Omidvar, E. Marx, H. Xin, Phys. Chem. Chem. Phys. 2018, 20, 6055-6059.

[43] V. I. Pârvulescu, P. Grange, B. Delmon, Catal. Today 1998, 46, 233-316.

[44] P. Xie, W. Ji, Y. Li, C. Zhang, Catal. Sci. Technol. 2021, 11, 374-391.

[45] F. Garin, Appl. Catal. A: Gen. 2001, 222, 183-219.

[46] S. Roy, M. S. Hegde, G. Madras, Appl. Energy 2009, 86, 2283-2297.

[47] M. Haneda, H. Hamada, C. R. Chim. 2016, 19, 1254-1265.

[48] H. Falsig, J. Shen, T. S. Khan, W. Guo, G. Jones, S. Dahl, T. Bligaard, Top Catal 2014, 57, 8088.

[49] M. M. Montemore, C. F. Nwaokorie, G. O. Kayode, Catal. Sci. Technol. 2020, 10, 4467-4476.

[50] K. J. Lim, D. G. Löffler, M. Boudart, J. Catal. 1986, 100, 158-166.

[51] J. E. Sutton, D. G. Vlachos, J. Catal. 2016, 338, 273-283.

[52] S. Wang, B. Temel, J. Shen, G. Jones, L. C. Grabow, F. Studt, T. Bligaard, F. Abild-Pedersen, C. H. Christensen, J. K. Nørskov, Catal Lett 2011, 141, 370-373.

[53] J. K. Nørskov, F. Studt, F. Abild-Pedersen, T. Bligaard, in Fundamental Concepts in Heterogeneous Catalysis, John Wiley \& Sons, 2014, pp. 97-113.

[54] H. Xin, A. Vojvodic, J. Voss, J. K. Nørskov, F. Abild-Pedersen, Phys. Rev. B 2014, 89, 115114.

[55] T. Bligaard, K. Honkala, A. Logadottir, J. K. Nørskov, S. Dahl, C. J. H. Jacobsen, J. Phys. Chem. B 2003, 107, 9325-9331.

[56] C. T. Campbell, ACS Catal. 2017, 7, 2770-2779.

[57] C. Stegelmann, A. Andreasen, C. T. Campbell, J. Am. Chem. Soc. 2009, 131, 8077-8082.

[58] C. T. Campbell, Top Catal 1994, 1, 353-366.

[59] C. T. Campbell, J. R. V. Sellers, Faraday Discuss. 2013, 162, 9-30.

[60] T. Furusawa, K. Aika, Bull. Chem. Soc. Jpn. 2000, 73, 795-800.

[61] X. Wang, S. M. Sigmon, J. J. Spivey, H. H. Lamb, Catal Today 2004, 96, 11-20.

[62] H. Falsig, T. Bligaard, J. Rass-Hansen, A. L. Kustov, C. H. Christensen, J. K. Nørskov, Top Catal 2007, 45, 117-120.

[63] P. Majumdar, J. Greeley, Phys. Rev. Materials 2018, 2, 045801.

[64] Z. Xu, J. R. Kitchin, J. Phys. Chem. C 2014, 118, 25597-25602.

[65] L. M. Herder, J. M. Bray, W. F. Schneider, Surf. Sci. 2015, 640, 104-111.

[66] M. Jørgensen, H. Grönbeck, ACS Catal. 2017, 5054-5061.

[67] M. Jørgensen, H. Grönbeck, ACS Catal. 2019, 9, 8872-8881.

[68] P. Giannozzi, S. Baroni, N. Bonini, M. Calandra, R. Car, C. Cavazzoni, D. Ceresoli, G. L.

Chiarotti, M. Cococcioni, I. Dabo, A. D. Corso, S. de Gironcoli, S. Fabris, G. Fratesi, R. Gebauer, U. Gerstmann, C. Gougoussis, A. Kokalj, M. Lazzeri, L. Martin-Samos, N. Marzari, F. Mauri, R. Mazzarello, S. Paolini, A. Pasquarello, L. Paulatto, C. Sbraccia, S. Scandolo, G. Sclauzero, A. P. 
Seitsonen, A. Smogunov, P. Umari, R. M. Wentzcovitch, J. Phys.: Condens. Matter 2009, 21, 395502.

[69] A. H. Larsen, J. J. Mortensen, J. Blomqvist, I. E. Castelli, R. Christensen, M. Dullak, J. Friis, M. N. Groves, B. Hammer, C. Hargus, E. D. Hermes, P. C. Jennings, P. B. Jensen, J. Kermode, J. R. Kitchin, E. L. Kolsbjerg, J. Kubal, K. Kaasbjerg, S. Lysgaard, J. B. Maronsson, T. Maxson, T. Olsen, L. Pastewka, A. Peterson, C. Rostgaard, J. Schiøtz, O. Schütt, M. Strange, K. S. Thygesen, T. Vegge, L. Vilhelmsen, M. Walter, Z. Zeng, K. W. Jacobsen, J. Phys.: Condens. Matter 2017, 29, 273002.

[70] B. Hammer, L. B. Hansen, J. K. Nørskov, Phys. Rev. B 1999, 59, 7413-7421.

[71] H. J. Monkhorst, J. D. Pack, Phys. Rev. B 1976, 13, 5188-5192.

[72] D. Vanderbilt, Phys. Rev. B 1990, 41, 7892-7895.

[73] L. Bengtsson, Phys. Rev. B 1999, 59, 12301-12304.

[74] K. T. Winther, M. J. Hoffmann, J. R. Boes, O. Mamun, M. Bajdich, T. Bligaard, Sci. Data. 2019, 6,75 . 
Table of contents graphics:
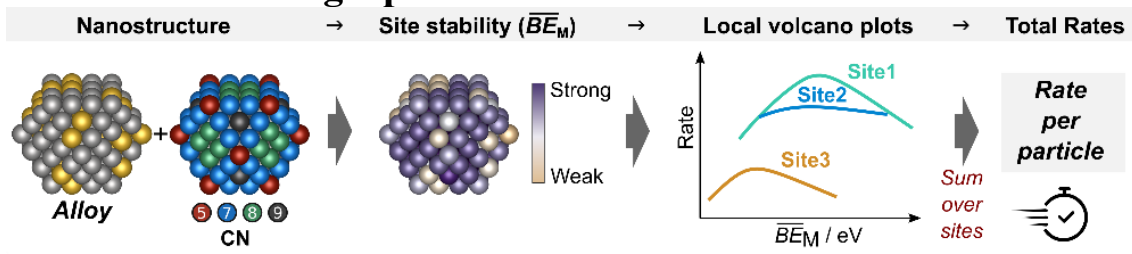

Table of contents text:

Using the site stability $\left(\overline{B E}_{M}\right)$ as unifying descriptor for activity and durability, we identify design principles for catalytically active sites. By modifying the local structure and alloying, we propose that the catalytic activity in NO decomposition over Pt-based nanoparticles can be enhanced by orders of magnitude. Our methods are general for metal active sites, and pave the way towards the inverse design of tailor-made heterogeneous catalysts. 